\title{
Advances in targeting the vacuolar proton-translocating ATPase (V-ATPase) for anti-fungal therapy
}

\author{
Summer R. Hayek ${ }^{1}$, Samuel A. Lee ${ }^{2,3}$ and Karlett J. Parra ${ }^{1}$ * \\ ${ }^{1}$ Department of Biochemistry and Molecular Biology, School of Medicine, University of New Mexico Health Sciences Center, Albuquerque, NM, USA \\ 2 Department of Internal Medicine, School of Medicine, University of New Mexico Health Sciences Center, Albuquerque, NM, USA \\ ${ }^{3}$ Section of Infectious Diseases, New Mexico Veterans Healthcare System, Albuquerque, NM, USA
}

\section{Edited by:}

George Tegos, University of New Mexico, USA

\section{Reviewed by:}

Partha Krishnamurthy, University of Kansas Medical Center, USA George Diallinas, University of Athens, Greece

\section{${ }^{*}$ Correspondence:}

Karlett J. Parra, Department of Biochemistry and Molecular Biology, School of Medicine, University of New Mexico Health Sciences Center MS08 4670, Albuquerque, 87131

New Mexico, USA

e-mail: kjparra@salud.unm.edu
Vacuolar proton-translocating ATPase (V-ATPase) is a membrane-bound, multi-subunit enzyme that uses the energy of ATP hydrolysis to pump protons across membranes. V-ATPase activity is critical for $\mathrm{pH}$ homeostasis and organelle acidification as well as for generation of the membrane potential that drives secondary transporters and cellular metabolism. V-ATPase is highly conserved across species and is best characterized in the model fungus Saccharomyces cerevisiae. However, recent studies in mammals have identified significant alterations from fungi, particularly in the isoform composition of the 14 subunits and in the regulation of complex disassembly. These differences could be exploited for selectivity between fungi and humans and highlight the potential for V-ATPase as an anti-fungal drug target. Candida albicans is a major human fungal pathogen and causes fatality in $35 \%$ of systemic infections, even with anti-fungal treatment. The pathogenicity of $C$. albicans correlates with environmental, vacuolar, and cytoplasmic $\mathrm{pH}$ regulation, and V-ATPase appears to play a fundamental role in each of these processes. Genetic loss of V-ATPase in pathogenic fungi leads to defective virulence, and a comprehensive picture of the mechanisms involved is emerging. Recent studies have explored the practical utility of V-ATPase as an anti-fungal drug target in C. albicans, including pharmacological inhibition, azole therapy, and targeting of downstream pathways. This overview will discuss these studies as well as hypothetical ways to target V-ATPase and novel high-throughput methods for use in future drug discovery screens.

Keywords: fungal V-ATPase, vacuolar proton pump, vacuolar acidification, pH homeostasis, $C$. albicans virulence, anti-fungal target

\section{V-ATPase PUMPS: STRUCTURE-FUNCTION AND MECHANISM OF CATALYSIS}

VACUOLAR H $^{+}$-ATPase (V-ATPase) PUMPS ARE LARGE MULTI-SUBUNIT MOLECULAR MOTORS THAT COUPLE ACTIVE TRANSPORT OF PROTONS WITH ATP HYDROLYSIS TO ACIDIFY INTRACELLULAR COMPARTMENTS

V-ATPase pumps generate and sustain the distinctive organelle $\mathrm{pH}$ gradient of the endomembrane system that is necessary for Golgi, endosomal, vacuolar, and lysosomal functions (Kane, 2006; Forgac, 2007). Redistribution of protons from the cytosol to the lumen of acidic organelles by V-ATPase pumps is essential for organelle $\mathrm{pH}$ homeostasis. In fungi, V-ATPase also contributes to cytosolic pH regulation (Martinez-Munoz and Kane, 2008). Genetic and pharmacologic inactivation of V-ATPase pumps alters intracellular and extracellular $\mathrm{pH}$. It disturbs numerous cellular processes including protein processing and sorting, protein secretion, receptor-mediated endocytosis, vesicular membrane trafficking, zymogen activation, and autophagy (Kane, 2006; Forgac, 2007).

The vast majority of structural and mechanistic data on eukaryotic V-ATPases available have been collected in Saccharomyces cerevisiae. V-ATPase proton transport requires structural and functional coupling of a peripheral domain $\left(\mathrm{V}_{1}\right)$ with a membrane-embedded domain $\left(\mathrm{V}_{\mathrm{o}}\right.$; Figure 1). Coupling involves an intricate mechanism that uses relative rotation of subunits in $V_{1}$ and $V_{\mathrm{o}}$ (Sun-Wada etal., 2003; Forgac, 2007). During catalysis, hydrolysis of ATP within the protuberant structure of $V_{1}$ drives rotation of a central stalk (the rotor's shaft) located near the catalytic sites. The rotating central stalk is connected to a hydrophobic ring of proteolipid-like subunits in $V_{\mathrm{o}}$ (c-ring). During rotation, each subunit of the c-ring has one essential glutamate residue that accepts a proton from the cytosol and transfers it to the organelle's lumen against a concentration gradient.

Important changes occurred as V-ATPase evolved from S. cerevisiae to humans. For example, the c-ring of all fungal species contains a combination of three subunits $\left(\mathrm{V}_{\mathrm{o}} \mathrm{c}, \mathrm{V}_{\mathrm{o}} \mathrm{c}^{\prime}, \mathrm{V}_{\mathrm{o}} \mathrm{c}^{\prime \prime}\right)$ whereas mammalian c-rings lack the $\mathrm{V}_{\mathrm{o}} \mathrm{c}^{\prime}$ subunit. Phylogenic analysis by Finnigan et al. (2012) suggests that the fungal $V_{o} c$ and $\mathrm{V}_{\mathrm{o}} \mathrm{c}^{\prime}$ subunits evolved from a gene duplication in an ancestral gene that was common to all fungal species. Intriguingly, the functions of $\mathrm{V}_{\mathrm{o}} \mathrm{c}$ and $\mathrm{V}_{\mathrm{o}} \mathrm{c}^{\prime}$ in fungi, particularly the binding capabilities of each subunit, appear to have degenerated from the common ancestor (Finnigan et al., 2012). This example of constructive neutral evolution suggests that the complexity of the V-ATPase machine may have been driven in part by loss-of-function processes (Doolittle, 2012). 
In general, the complexity of V-ATPase increased as species evolved further from fungi. Mammals developed multiple tissue and membrane specific isoforms for most $\mathrm{V}_{1}$ and $\mathrm{V}_{\mathrm{o}}$ subunits (Figure 1; Marshansky and Futai, 2008; Sun-Wada and Wada, 2010; Toei et al., 2010). In contrast, only one subunit ( $\left.\mathrm{V}_{\mathrm{o}} \mathrm{a}\right)$ of $\mathrm{V}$ ATPase in budding yeast species such as $S$. cerevisiae has functional homologues (Manolson et al., 1994), and non-budding fungi such as Neurospora crassa contain only single subunit isoforms (Chavez et al., 2006).

The sequence conservation between human and S. cerevisiae subunits is $31-41 \%$ identity and $51-60 \%$ similarity, depending on the subunit and isoform (Rahman et al., 2013). This relatively low sequence conservation may explain differences in binding affinity between V-ATPase subunits from human (Rahman et al., 2013) and S. cerevisiae (Oot and Wilkens, 2012); it may also fine tune VATPase activity and determine regulatory mechanisms in response to diverse cellular signals and environments. Although information describing the topological arrangements of the human $\mathrm{V}_{1} \mathrm{~V}_{\mathrm{o}}$ complex is not available, the V-ATPase overall structure and its sophisticated mechanism of rotational catalysis are likely conserved from S. cerevisiae.

This review focuses on fungal V-ATPases with an emphasis on the human fungal pathogen Candida albicans. We summarize our current understanding of the roles of V-ATPase in pathogenicity as well as its antifungal drug targeting potential. Because V-ATPase subunit structure and composition, assembly and regulation, and multiple downstream cellular functions are best studied in the fungus S. cerevisiae (Kane, 2006, 2007), we also refer to $S$. cerevisiae and other fungi throughout this review.

\section{Candida albicans IS THE PRIMARY HUMAN FUNGAL PATHOGEN}

Candida albicans is the most frequently diagnosed fungal pathogen and is the fourth leading cause of hospital-acquired bloodstream infections in North America (Klotz et al., 2007). C. albicans is normally a harmless commensal in the oral cavity, digestive tract, and genital region of healthy people but is also associated with superficial infections. C. albicans can enter the bloodstream following tissue damage or the formation of fungal biofilms on medical implants, leading to sepsis, and organ failure. These severe cases of systemic candidiasis are most common in patients undergoing immunosuppressive therapy or that are otherwise immunocompromised (Pfaller and Diekema, 2007). Critically, patient mortality rates can reach $35 \%$ even with anti-fungal treatment (Horn et al., 2009).

Invasive infection due to $C$. albicans is a multifactorial process that relies on numerous virulence factors to control pathogenesis. C. albicans can exist as either a unicellular yeast or a filamentous hyphae. This morphological dimorphism contributes to virulence, as the yeast form is considered nonpathogenic while the hyphal form induces damage and invasion of host tissue (Sudbery, 2011). C. albicans also secretes serine aspartyl proteinases and lipases that are involved in nutrient acquisition, host cell degradation, and immune evasion (Naglik et al., 2003). Other C. albicans virulence pathways include iron acquisition from hemoglobin, protection
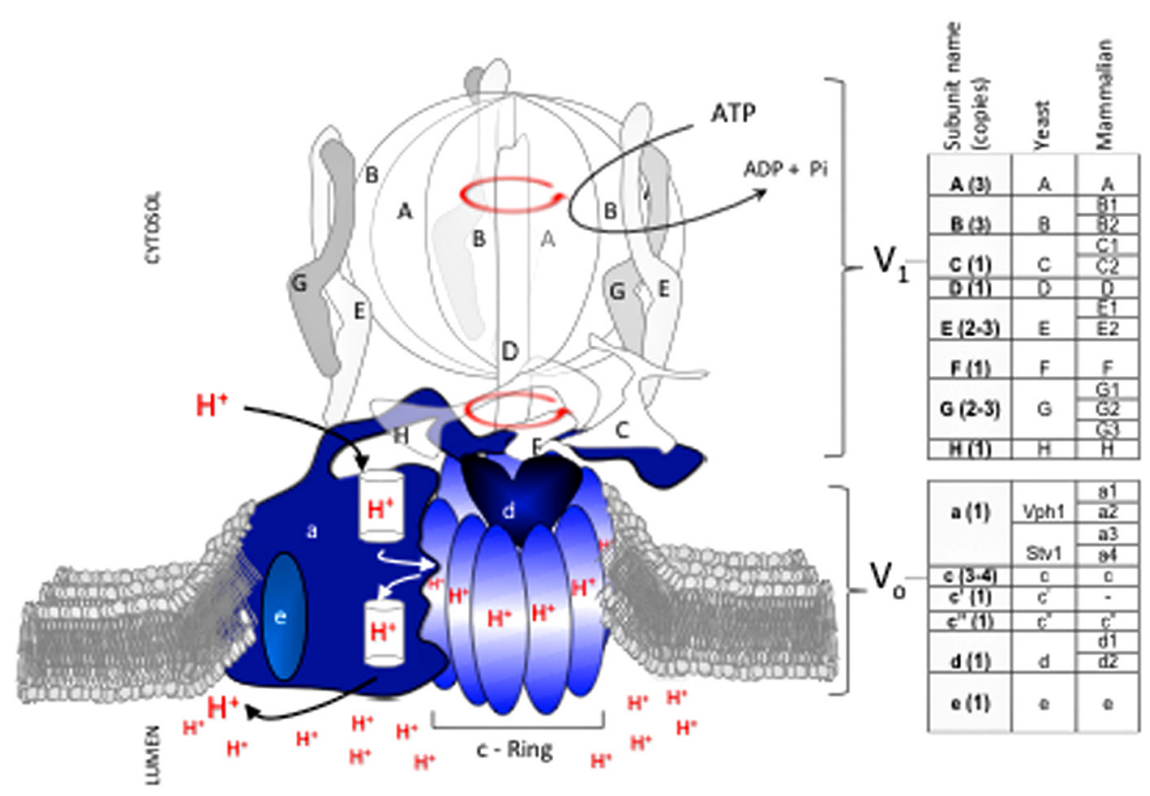

FIGURE 1 | V-ATPase subunit composition and mechanism of catalysis. The V-ATPase proton pump acidifies the lumen of organelles in the endomembrane system of all eukaryotic cells. V-ATPase has 14 subunits that form two domains, $V_{1}$ and $V_{0} . V_{1}$ (clear and gray subunits, $A_{3} B_{3} C D E_{3} F G_{3} H$ ) hydrolyzes ATP at the cytosolic side of the membrane, and $V_{0}$ (blue subunits, $\mathrm{ac}_{3}-4 \mathrm{C}^{\prime} \mathrm{c}^{\prime \prime} \mathrm{de}$ ) translocates protons. Transport of protons against a concentration gradient entails a rotational mechanism. Hydrolysis of 3 ATP in the $V_{1} A$ catalytic subunits drives rotation of a shaft (subunits $D, F$ ) that penetrates $V_{1}$ and is bound to a rotating proteolipid ring structure in $V_{O}$ formed by subunits $\mathrm{C}_{(3-4)} \mathrm{C}^{\prime} \mathrm{C}^{\prime \prime}$ (c-ring) that together with subunit $\mathrm{V}_{\mathrm{O}}$ a forms the path for proton transport. With the exception of subunit $V_{0} a$, which exists in two isoforms (Vph1p and Stv1p), each V-ATPase subunit is encoded by a single gene in fungi. In contrast, multiple isoforms (two to four) exist for most subunits of the mammalian V-ATPase. Subunit $V_{0} c^{\prime}$ is absent in mammals. 
against reactive oxygen species, expression of adhesion molecules, and formation of biofilms. Together, these pathways facilitate host cell invasion and protect against the host immune response (Karkowska-Kuleta et al., 2009).

\section{Candida albicans PATHOGENICITY CORRELATES WITH pH REGULATION, SUGGESTING THAT V-ATPase MAY PLAY A FUNDAMENTAL ROLE IN FUNGAL VIRULENCE Candida albicans VIRULENCE IS REGULATED BY pH AT THE EXTRACELLULAR, VACUOLAR, AND CYTOPLASMIC LEVEL}

Extracellular $\mathrm{pH}$ controls the morphological dimorphism of $C$. albicans. The non-pathogenic yeast form of the fungus grows preferentially under acidic environmental conditions while increasing extracellular $\mathrm{pH}$ triggers hyphal growth and increased virulence (Sudbery, 2011). C. albicans has developed mechanisms that allow it to rapidly respond to environmental $\mathrm{pH}$ changes, and mutants lacking the ability to sense extracellular $\mathrm{pH}$ display reduced virulence (Davis, 2009).

Vacuolar $\mathrm{pH}$ is important for numerous aspects of C. albicans physiology and virulence. Preservation of a proton gradient across the vacuolar membrane is critical for general cellular metabolism, including receptor-mediated endocytosis, intracellular membrane trafficking, pro-hormone processing, protein degradation, uptake of small molecules, and storage and detoxification of metabolites and ions (Veses et al., 2008). Additionally, both activation and secretion of the proteinase and lipase virulence factors and activity of the enzymes themselves require optimal vacuolar $\mathrm{pH}$ (Naglik et al., 2003). Numerous C. albicans mutants that display abnormal vacuolar alkalinization also display reduced filamentation and defective in vivo virulence (Bruckmann etal., 2000; Jia et al., 2002; Eck et al., 2005; Poltermann et al., 2005; Palanisamy et al., 2010; Zhang et al., 2010; Patenaude et al., 2013; Rane et al., 2013). The azole class of anti-fungal drugs also functions in part through disruption of vacuolar acidification (Zhang et al., 2010).

Finally, cytoplasmic $\mathrm{pH}$ contributes to filament formation during C. albicans virulence. Germ tube formation, the precursor step to hyphal formation, requires alkalinization of the cytoplasm (Stewart etal., 1988). In fungi, cytosolic pH is regulated via the Pmalp plasma membrane proton transporter, which pumps protons out of the cell and into the extracellular space to maintain a neutral-to-alkaline cytosol and an acidic extracellular environment (Monk etal., 1991). The importance of Pmalp activity and cytosolic alkalinization in C. albicans virulence is illustrated by studies showing that Pmalp activity and expression is upregulated during filamentation (Kaur and Mishra, 1991; Monk et al., 1993). Furthermore, C. albicans mutants that cannot properly alkalinize their cytosol in response to filamentation cues are avirulent (Stewart et al., 1988, 1989; Mahanty et al., 1990).

The central importance of $\mathrm{pH}$ regulation in C. albicans virulence makes V-ATPase an attractive target for anti-fungal therapy (Parra, 2012). In addition to its critical role in vacuolar $\mathrm{pH}$ homeostasis (Kane, 2006; Tarsio et al., 2011), V-ATPase is a known regulator of Pmalp activity in S. cerevisiae. V-ATPase mutants display abnormally acidified cytosol due to a lack of properly localized or functional Pmalp (Perzov et al., 2000; Martinez-Munoz and
Kane, 2008; Huang and Chang, 2011). Together, these data suggest that V-ATPase may contribute to numerous virulence pathways in C. albicans, including vacuolar function and during the germ tubeto-hyphae morphological transition. Next, we summarize studies that have examined the role of V-ATPase in fungal pathogenesis using genetic loss-of-function studies.

\section{GENETIC LOSS OF V-ATPase IN PATHOGENIC FUNGI AFFECTS VIRULENCE GENETIC STUDIES IN VARIOUS PATHOGENIC FUNGI HAVE ESTABLISHED A LINK BETWEEN V-ATPase ACTIVITY, VACUOLAR ACIDIFICATION, AND FUNGAL VIRULENCE}

Hilty et al. (2008) removed VMA1 (the $\mathrm{V}_{1} \mathrm{~A}$ subunit of V-ATPase) from the Histoplasma capsulatum genome and demonstrated that $\mathrm{V}_{1} \mathrm{~A}$ is required for iron sequestration, replication in macrophages, and growth as a mold. The vmal mutants were also avirulent in a mouse model of histoplasmosis (Hilty et al., 2008). In Cryptococcus neoformans, loss of the $\mathrm{V}_{\mathrm{o}}$ a subunit via deletion of $\mathrm{VPH} 1$ leads to defective production of capsule, laccase, and urease, three virulence factors required for $C$. neoformans infectivity. These $v p h 1$ mutants also displayed defective in vivo virulence in a murine model of meningo-encephalitis (Erickson et al., 2001). These findings lend credence to the idea that V-ATPase plays a critical role in the maintenance of fungal virulence.

In contrast, studies in Aspergillus fumigatus suggest that host V-ATPase plays an important protective role during immune defense against fungal pathogens. A. fumigatus is the primary causative agent of life-threatening invasive bronchopulmonary aspergillosis. While host V-ATPase is critical for phagolysosomal acidification and pathogen killing under non-pathogenic conditions, infective variants of $A$. fumigatus prevent acidification of phagolysosomes and allow for A. fumigatus germination and immune escape (Ibrahim-Granet et al., 2003). Notably, work by Slesiona et al. demonstrated that less virulent Aspergillus variants can be made virulent via pharmacological inhibition of host V-ATPase resulting in loss of phagolysosomal acidification (Slesiona etal., 2012). These findings suggest that fungal pathogens may inactivate host V-ATPase pathways during immune evasion and suggest that the balance of pathogen versus host V-ATPase activity is critical for determining virulence.

Studies specifically examining virulence in C. albicans have further solidified the importance of V-ATPase in this process. In the iron-deplete conditions of host tissue, C. albicans must extract iron from hemoglobin for survival. Weissman et al. (2008) demonstrated that null mutants of vma11 (subunit $\mathrm{V}_{\mathrm{o}} \mathrm{c}^{\prime}$ ) are deficient in iron acquisition. Upon loss of VMA7 (subunit $\mathrm{V}_{1} \mathrm{~F}$ ) in C. albicans, Poltermann et al. (2005) noted defects in in vitro filamentation and in vivo virulence during systemic candidiasis. The authors further connected these defects to biochemical phenotypes including vacuolar alkalinization, $\mathrm{pH}$-dependent growth, and sensitivity to metal ions (Poltermann et al., 2005). Recently, our laboratories demonstrated that inducible loss of VMA3 (subunit $\mathrm{V}_{\mathrm{o}} \mathrm{c}$ of the cring) in C. albicans results in alkaline vacuoles with fission defects, leading to reduced protease and lipase secretion, defective filamentation, and ineffective macrophage killing (Rane et al., 2013). 
Unpublished studies from our laboratories have yielded similar phenotypes following the inducible loss of VMA2 (subunit $\mathrm{V}_{1} \mathrm{~B}$ ).

In $C$. albicans, the $\mathrm{V}_{\mathrm{o}}$ a subunit is the only subunit within the enzyme complex that is encoded by multiple isoforms: VPH1 localizes V-ATPase to vacuoles while STV1 traffics V-ATPase to the Golgi and endocytic organelles (Patenaude et al., 2013; Raines et al., 2013). This difference was recently exploited to study the contribution of organelle-specific V-ATPase to C. albicans virulence. Both our laboratories and Patenaude et al. (2013) showed that loss of VPH1 but not STV1 leads to vacuolar alkalinization, abnormal vacuolar morphology, and defective metal ion sequestration (Raines et al., 2013). Our study noted that although these biochemical defects contributed to reduced protease and lipase secretion, the $v p h 1$ mutants displayed only modest filamentation defects and wild-type levels of biofilm formation and macrophage killing (Raines et al., 2013). Our results suggest that vacuolar acidification is dispensable during certain $C$. albicans virulence pathways. Furthermore, Stvlp-containing V-ATPase complexes may play novel roles in pathogenesis (Figure 2). In contrast, the $v p h 1$ mutant cells used in the Patenaude et al. (2013) study displayed defective filamentation, reduced damage to epithelial and macrophage host cells, and avirulence during systemic in vivo infection. These results suggest that vacuolar acidification is essential for all forms of C. albicans virulence. The explanation for these disparate results remains to be determined but likely centers around differences in strain background or methodology. Nonetheless, these studies raise the fascinating possibility that organelle-specific V-ATPase activity can be modulated to control specific C. albicans virulence pathways.

\section{CURRENT UTILITY OF V-ATPase AS AN ANTI-FUNGAL DRUG TARGET}

The recent emergence of multidrug resistant strains of C. albicans has made the development of novel classes of anti-fungal drugs paramount (Hameed and Fatima, 2013). V-ATPase is an attractive target for drug discovery, given the numerous lines of genetic evidence supporting a critical role for V-ATPase in C. albicans virulence. We next consider the feasibility of VATPase as an anti-fungal drug target via an overview of three currently available methods that utilize V-ATPase-related mechanisms: pharmacological inhibition of V-ATPase, azole therapy, and Pmalp inhibition.

\section{PHARMACOLOGICAL INHIBITION OF V-ATPase}

V-ATPase inhibitors have been used for over 20 years to study the function and mechanism of V-ATPase activity in organisms ranging from fungi to humans. As of 2009, eight types of V-ATPase inhibitors had been described, including the best characterized plecomacrolide class (Huss and Wieczorek, 2009). The plecomacrolides include bafilomycin and concanamycin, which are antibiotics that bind to the $\mathrm{V}_{\mathrm{o}} \mathrm{c}$ subunit of V-ATPase to prevent c-ring rotation and interfere with ATP hydrolysis and proton transport simultaneously (Bowman et al., 1988; Drose et al., 1993). Notably, both bafilomycin and concanamycin have been shown to inhibit V-ATPase from C. albicans (Calvert and Sanders, 1995; Chan et al., 2012). However, none of the molecules belonging to the aforementioned eight classes of V-ATPase inhibitors are currently used to treat $C$. albicans infection in a clinical setting; many cannot differentiate between the fungal target and mammalian

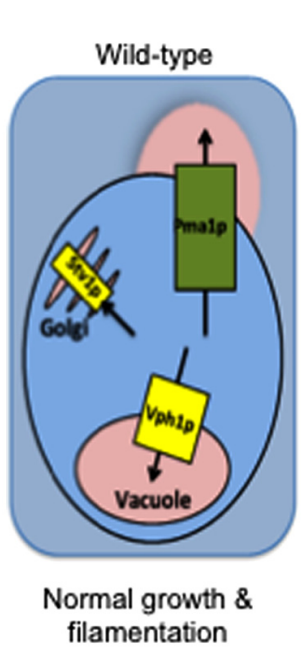

filamentation

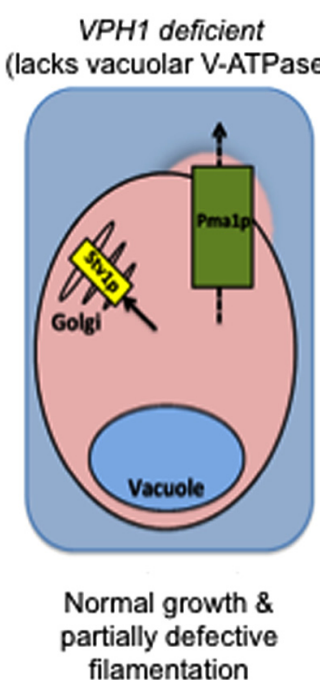

FIGURE 2 | V-ATPase in non-vacuolar organelles (Stv1p-containing complexes) plays a yet unknown role in $\boldsymbol{C}$. albicans virulence. Wild-type: When functional, V-ATPase-mediated acidification of vacuoles, Golgi, and secretory vesicles maintains organelle $\mathrm{pH}$ and supports traffic of Pma1 $\mathrm{p}$ to the cell surface for proton efflux and maintenance of an alkaline cytosol. VPH1 deficient: $C$. albicans grows normally at neutral $\mathrm{pH}$ when only Vph1p-containing V-ATPase complexes (vacuolar membrane) are missing. Only modest filamentation defects are obvious, despite the concomitant vacuolar alkalinization and defective Pma1p activity (Raines et al., 2013 and unpublished results). VMA3 deficient: Vacuolar alkalinization and defective Pma1p activity occur at levels equal to that of VPH1 deficient cells when all V-ATPase function is missing (Stv1p- and Vph1-containing V-ATPase complexes; Rane et al., 2013 and unpublished results). However, VMA3 deficient $C$. albicans exhibits growth defects at neutral $\mathrm{pH}$ and severely reduced filamentation under these conditions. We therefore hypothesize that the presence of Stv1p-containing V-ATPase in non-vacuolar organelles maintains virulence in the face of defective vacuolar and cytoplasmic $\mathrm{pH}$ homeostasis. Pink = acidic/acidified, blue = alkaline/alkalinized 
host V-ATPase (Bowman and Bowman, 2005; Huss and Wieczorek, 2009).

Recently, our laboratory developed a high-throughput screening method to identify new V-ATPase inhibitors in S. cerevisiae (Chan et al., 2012). In this method, S. cerevisiae cells are transformed with the gene for pHluorin, a pH-sensitive version of GFP (Brett et al., 2005). The fluorescence intensity of pHluorin can be used to identify molecules that acidify the cytosol of $S$. cerevisiae following V-ATPase inhibition. We screened the Prestwick Chemical Library (a collection of 1120 off-patent drug compounds) using this method and identified alexidine dihydrochloride and thonzonium bromide as novel and specific V-ATPase inhibitors (Chan etal., 2012). Cells treated with these drugs showed a VATPase-specific $\mathrm{pH}$-sensitive growth phenotype ( vma phenotype) exclusive of fungi species. These inhibitors belong to a unique class of V-ATPase uncouplers that inhibit proton transport without affecting ATP hydrolysis. We also confirmed that disulfiram, a compound identified previously by a high-throughput screening method that identifies drugs that alkalinize vacuoles (Johnson et al., 2010), inhibits V-ATPase activity via the pHluorin screening method.

Alexidine dihydrochloride, thonzonium bromide, and disulfiram also inhibit V-ATPase in isolated C. albicans vacuoles. Alexidine dihydrochloride and thonzonium bromide also cause general cellular toxicity in intact C. albicans cells (Chan et al., 2012). These data suggest that these pre-existing drugs could be repurposed as anti-fungal therapies. Indeed, the majority of compounds in the Prestwick Chemical Library have known safety and toxicity profiles (Wermuth, 2004), making drug repurposing a less daunting task than de novo drug discovery.

\section{AZOLE THERAPY}

Azoles are a class of antifungal agents that are commonly used to treat fungal infections in the clinical setting. Azoles inhibit C-14 $\alpha$-demethylase, an enzyme necessary for the conversion of lanosterol to ergosterol, an essential component of fungal membranes (Chapman et al., 2008). At least four ergosterol biosynthesis genes (ERG3, ERG4, ERG5, and ERG11) are affected by azoles. The general membrane disruption resulting from azole treatment alters the activity of several membrane-bound transporters and channels including those associated with the transport of amino acids and other nutrients, chitin synthesis, and mitochondrial oxidation (Vanden Bossche, 1985; Barrett-Bee et al., 1991; Georgopapadakou and Walsh, 1996).

For some time, it was assumed that azoles caused fungal toxicity primarily through damage to the cell membrane resulting in cell permeability and lysis (Chapman et al., 2008). However, recent work has uncovered a critical role for ergosterol in V-ATPase function. In S. cerevisiae, mutants defective in ergosterol biosynthesis display alkaline vacuoles and the vma phenotype (Zhang et al., 2010). Surprisingly, the V-ATPase holoenzyme is properly assembled at the vacuolar membrane despite ergosterol depletion. The mutant phenotypes instead appear to result from reduced ATP hydrolysis and proton pumping within the V-ATPase. Fluconazole treatment of $C$. albicans cells also results in alkaline vacuoles (Zhang et al., 2010), further solidifying that azoles function in part through inhibition of V-ATPase.
These studies by Zhang etal. (2010) have identified a new regulatory pathway involved in V-ATPase function as well as elucidated a novel mechanism underlying azole toxicity in C. albicans. From a therapeutic standpoint, these studies provide proof-ofprinciple that anti-V-ATPase agents could work in the clinical setting. Importantly, drugs targeting V-ATPase may act mechanistically similar to azoles while bypassing the multidrug resistance that has plagued current anti-fungal therapies.

\section{Pma1p INHIBITION}

Given the interplay between V-ATPase and Pmalp in S. cerevisiae (Perzov et al., 2000; Martinez-Munoz and Kane, 2008; Huang and Chang, 2011) and C. albicans (unpublished results), antifungal therapies that rely on Pmalp inhibition may affect pathways downstream of V-ATPase function. Indeed, Bowman et al. (1997) demonstrated that 64 of 66 mutations that rescued concanamycin A-mediated V-ATPase inhibition in N. crassa were localized to the PMA1 gene. The synthetic organoselenium compound ebselen is a known inhibitor of Pmalp (Chan et al., 2007; Billack et al., 2009). Ebselen exhibits anti-fungal activity against wild-type strains of C. albicans (Soteropoulos etal., 2000; Bouhafs and Jarstrand, 2002; Wojtowicz et al., 2004) as well as fluconazole-resistant strains (Billack et al., 2009). These results again demonstrate the precedence that targeting components of the V-ATPase pathway, even via a downstream element such as Pmalp, can circumvent the drug resistance that has developed against presently available therapies.

\section{HYPOTHETICAL APPROACHES FOR TARGETING V-ATPase THE DEVELOPMENT OF NEW ANTI-FUNGAL TREATMENTS AGAINST V-ATPase MUST INVOLVE NOVEL STRATEGIES FOR IDENTIFYING THERAPEUTIC TARGETS}

Selectivity for fungal pathogens over innocuous host tissue is one critical targeting factor to consider. The $\mathrm{V}_{\mathrm{o}} \mathrm{c}^{\prime}$ subunit of V-ATPase, encoded by VMA11, is a promising candidate target (Parra, 2012). The $V_{o} c^{\prime}$ subunit is found specifically in fungi and lacks a mammalian homolog (Figure 1). Promisingly, previous studies have demonstrated that C. albicans null mutants of vma11 do not properly acquire iron from hemoglobin, a critical virulence factor within host tissue (Weissman et al., 2008). Future structural studies of the $\mathrm{V}_{\mathrm{o}} \mathrm{c}^{\prime}$ subunit will aid in the rational design of a fungal-specific anti-V-ATPase drug.

Most fungal V-ATPase subunits are encoded by a single gene; the $\mathrm{V}_{\mathrm{o}}$ a subunit is the only subunit encoded by two isoforms. Mammalian V-ATPases are strikingly different, with seven different subunits displaying isoform variation (Jefferies et al., 2008; Figure 1). This drastic difference between fungi and mammals could be exploited for fungal-specific drug development. For example, the $\mathrm{V}_{1} \mathrm{C}$ subunit exists as three isoforms in mammals. If an inhibitor is designed to specifically target a domain of fungal $\mathrm{V}_{1} \mathrm{C}$ that is missing from one or more of the mammalian isoforms, all cellular V-ATPase activity will cease in the fungal pathogen while the non-targeted mammalian isoforms compensate and maintain V-ATPase activity in the host cell. This strategy will require high-resolution structures of all fungal and mammalian isoforms and is therefore a longer-range possibility. However, it remains a fascinating option for fungal-specific drug targeting. 
V-ATPase activity is regulated in part by reversible disassembly, the process by which the $V_{1}$ and $V_{o}$ sectors separate from one another to prevent ATP hydrolysis and proton transport (Kane and Parra, 2000; Kane, 2006). Reversible disassembly is triggered primarily by glucose deprivation, although extracellular $\mathrm{pH}$ and salt stress are thought to contribute to the process. In mammals, cell-specific regulatory pathways also control disassembly in a tissue-specific manner (Kane, 2012). Disassembly in C. albicans has not been studied, but conserved similarities between $S$. cerevisiae and mammals suggest $C$. albicans will utilize similar pathways. Notably, the $\mathrm{V}_{\mathrm{o}}$ a subunit and organelle environment appear to regulate disassembly in $S$. cerevisiae; Vphlp-containing complexes disassociate upon glucose deprivation while Stv1pcontaining complexes are less sensitive (Kawasaki-Nishi et al., 2001). In contrast, mammalian cells contain four $\mathrm{V}_{\mathrm{o}}$ a isoforms localized to different tissues. This suggests that differences in isoform structure and interaction, localization, or susceptibility to environmental cues could be exploited for fungal-specific drug development as described previously. Alternatively, indirectly targeting the regulator proteins involved in disassembly may allow for fungal-specific inhibition of V-ATPase activity (Kane, 2012).

Finally, Pmalp is a fungal-specific protein with no known mammalian homologs. Simultaneous targeting of both V-ATPase and Pmalp should therefore disrupt both vacuolar and cytoplasmic $\mathrm{pH}$ homeostasis in fungi versus vacuolar $\mathrm{pH}$ alone in mammalian cells. This dual approach may thereby prove more detrimental to the fungal pathogen than the host cell. This method is also advantageous in that it can be achieved either by creation of a novel V-ATPase/Pmalp dual inhibitor or by administration of an anti-fungal drug cocktail consisting of presently available therapies. Additionally, as Pmalp is a plasma membrane protein with access to the extracellular space, anti-Pmalp drugs may function regardless of accumulation within the fungal cell. This possibility is highly advantageous, as drug uptake often limits efficacy in fungi due to a lack of specific uptake systems.

\section{A NOVEL HIGH-THROUGHPUT METHOD FOR FUTURE V-ATPase DRUG DISCOVERY IN C. albicans}

Our need for anti-V-ATPase therapies grows increasingly critical as we improve our understanding of the function of V-ATPase in fungal virulence. However, direct screening for anti-V-ATPase drugs in C. albicans is typically hindered by the non-availability of high-throughput screening tools in the pathogenic fungi. We previously demonstrated the practical utility of measuring cytosolic $\mathrm{pH}$ as a surrogate for V-ATPase function in a high-throughput screen for inhibitors of S. cerevisiae V-ATPase (Chan et al., 2012). This method uses pHluorin, a pH-sensitive GFP construct that can be stably transformed, thereby avoiding expensive and timeconsuming single-use dyes. Recently, Ullah et al. (2013) were the first to successfully express pHluorin in a pathogenic fungus, $C$. glabrata. However, non-canonical codon use precludes the direct application of the existing pHluorin construct in C. albicans.

Our lab is currently working to create a pHluorin construct optimized for C. albicans (CapHluorin) that will enable easy, inexpensive, and high-throughput measurement of cytosolic $\mathrm{pH}$ for use in screening for V-ATPase inhibitors. Additionally, alkalinization of the cytoplasm is a general determinant of fungal virulence (Stewart et al., 1988), and CapHluorin-mediated drug screens may result in the rapid discovery of new anti-fungal therapies that function independently of V-ATPase. Previous studies in $S$. cerevisiae proved the feasibility of modifying pHluorin for targeting to specific cellular compartments such as the Golgi (Tarsio et al., 2011). CapHluorin will facilitate similar studies to examine the importance of organelle-specific V-ATPase function and $\mathrm{pH}$ homeostasis in C. albicans. Importantly, the CapHluorin construct optimized for non-canonical codon use will enable cytosolic $\mathrm{pH}$ measurements not just in C. albicans, but in all fungal species that utilize the CTG clade, including the human pathogens C. dubliniensis, C. tropicalis, C. parapsilosis, and C. lusitaniae (Papon et al., 2012).

\section{ACKNOWLEDGMENTS}

The authors gratefully acknowledge support, in whole or in part, from the National Institutes of Health (NIH), Bethesda, Maryland, USA, including Grants 5R01GM086495 (to Karlett J. Parra) and K12GM088021 (to Summer R. Hayek).

\section{KEY CONCEPTS}

VACUOLAR $\mathrm{H}^{+}$-ATPase (V-ATPase) PUMPS ARE LARGE MULTI-SUBUNIT MOLECULAR MOTORS THAT COUPLE ACTIVE TRANSPORT OF PROTONS WITH ATP HYDROLYSIS

V-ATPase activity is required for acidification of intracellular compartments and generates and sustains the $\mathrm{pH}$ gradient required for function of the endomembrane system organelles.

\section{Candida albicans IS THE MOST FREOUENTLY DIAGNOSED FUNGAL PATHOGEN}

Although it exists commensally with humans, it can cause life-threatening blood infections under optimal pathogenic conditions. C. albicans has developed resistance to many currently available anti-fungal drugs, and mortality rates can reach $35 \%$ even with proper treatment. There is therefore a dire need to develop new anti-fungal therapies.

\section{Candida albicans VIRULENCE IS REGULATED BY pH AT THE EXTRACELLULAR, VACUOLAR, AND CYTOPLASMIC LEVEL}

Extracellular $\mathrm{pH}$ triggers a morphological switch to the pathogenic form of the fungus. Vacuolar acidification allows for activation/secretion of virulence enzymes. Cytoplasmic alkalinization precedes the formation of germ tubes during C. albicans filamentation.

\section{GENETIC STUDIES IN VARIOUS PATHOGENIC FUNGI HAVE ESTABLISHED A LINK BETWEEN V-ATPase ACTIVITY, VACUOLAR ACIDIFICATION, AND FUNGAL VIRULENCE}

Studies in Histoplasma capsulatum, Cryptococcus neoformans, and Candida albicans have demonstrated that loss of all cellular VATPase leads to alkaline vacuoles and a host of in vitro and in vivo virulence defects.

\section{V-ATPase IS AN ATTRACTIVE TARGET FOR DRUG DISCOVERY}

Current anti-fungal therapies that involve the V-ATPase pathway include direct pharmacological inhibition of V-ATPase, the clinically prescribed azole class of drugs, and Pmalp inhibitors. 
However, the emergence of multidrug resistant strains of $C$. albicans makes additional drug discovery imperative.

\section{THE DEVELOPMENT OF NEW ANTI-FUNGAL TREATMENTS AGAINST V-ATPase MUST INVOLVE NOVEL STRATEGIES FOR IDENTIFYING THERAPEUTIC TARGETS}

Such strategies include targeting the fungal-specific $V_{o} c^{\prime}$ subunit, utilizing differences in isoform composition and complex disassembly between fungi and mammals, dual V-ATPase/Pmalp inhibition, and creation of a high-throughput screen in C. albicans.

\section{REFERENCES}

Barrett-Bee, K., Newboult, L., and Pinder, P. (1991). Biochemical changes associated with the antifungal action of the triazole ICI 153,066 on Candida albicans and Trichophyton quinckeanum. FEMS Microbiol. Lett. 63, 127-131. doi: 10.1111/j.1574-6968.1991.tb04517.x

Billack, B., Santoro, M., and Lau-Cam, C. (2009). Growth inhibitory action of ebselen on fluconazole-resistant Candida albicans: role of the plasma membrane H+-ATPase. Microb. Drug Resist. 15, 77-83. doi: 10.1089/mdr.2009.0872

Bouhafs, R. K., and Jarstrand, C. (2002). Effects of antioxidants on surfactant peroxidation by stimulated human polymorphonuclear leukocytes. Free Radic. Res. 36, 727-734. doi: 10.1080/10715760290032593

Bowman, E. J., and Bowman, B. J. (2005). V-ATPases as drug targets. J. Bioenerg. Biomembr. 37, 431-435. doi: 10.1007/s10863-005-9485-9489

Bowman, E. J., O’Neill, F. J., and Bowman, B. J. (1997). Mutations of pma-1, the gene encoding the plasma membrane $\mathrm{H}+$-ATPase of Neurospora crassa, suppress inhibition of growth by concanamycin A, a specific inhibitor of vacuolar ATPases. J. Biol. Chem. 272, 14776-14786. doi: 10.1074/jbc.272.23.14776

Bowman, E. J., Siebers, A., and Altendorf, K. (1988). Bafilomycins: a class of inhibitors of membrane ATPases from microorganisms, animal cells, and plant cells. Proc. Natl. Acad. Sci. U.S.A. 85, 7972-7976. doi: 10.1073/pnas.85.21.7972

Brett, C. L., Tukaye, D. N., Mukherjee, S., and Rao, R. (2005). The yeast endosomal $\mathrm{Na}+\mathrm{K}+/ \mathrm{H}+$ exchanger $\mathrm{Nhxl}$ regulates cellular $\mathrm{pH}$ to control vesicle trafficking. Mol. Biol. Cell 16, 1396-1405. doi: 10.1091/mbc.E04-11-0999

Bruckmann, A., Kunkel, W., Hartl, A., Wetzker, R., and Eck, R. (2000). A phosphatidylinositol 3-kinase of Candida albicans influences adhesion, filamentous growth and virulence. Microbiology 146(Pt. 11), 2755-2764.

Calvert, C. M., and Sanders, D. (1995). Inositol trisphosphate-dependent and independent $\mathrm{Ca} 2+$ mobilization pathways at the vacuolar membrane of Candida albicans. J. Biol. Chem. 270, 7272-7280. doi: 10.1074/jbc.270.13.7272

Chan, C. Y., Prudom, C., Raines, S. M., Charkhzarrin, S., Melman, S. D. De Haro, L. P., et al. (2012). Inhibitors of V-ATPase proton transport reveal uncoupling functions of tether linking cytosolic and membrane domains of V0 subunit a (Vph1p). J. Biol. Chem. 287, 10236-10250. doi: 10.1074/jbc.M111. 321133

Chan, G., Hardej, D., Santoro, M., Lau-Cam, C., and Billack, B. (2007). Evaluation of the antimicrobial activity of ebselen: role of the yeast plasma membrane $\mathrm{H}+-$ ATPase. J. Biochem. Mol. Toxicol. 21, 252-264. doi: 10.1002/jbt.20189

Chapman, S. W., Sullivan, D. C., and Cleary, J. D. (2008). In search of the holy grail of antifungal therapy. Trans. Am. Clin. Climatol. Assoc. 119, 197-215; discussion 215-196.

Chavez, C., Bowman, E. J., Reidling, J. C., Haw, K. H., and Bowman, B. J. (2006). Analysis of strains with mutations in six genes encoding subunits of the V-ATPase: eukaryotes differ in the composition of the V0 sector of the enzyme. J. Biol. Chem. 281, 27052-27062. doi: 10.1074/jbc.M603883200

Davis, D. A. (2009). How human pathogenic fungi sense and adapt to $\mathrm{pH}$ : the link to virulence. Curr. Opin. Microbiol. 12, 365-370. doi: 10.1016/j.mib.2009. 05.006

Doolittle, W. F. (2012). Evolutionary biology: a ratchet for protein complexity. Nature 481, 270-271. doi: 10.1038/nature10816

Drose, S., Bindseil, K. U., Bowman, E. J., Siebers, A., Zeeck, A., and Altendorf, K. (1993). Inhibitory effect of modified bafilomycins and concanamycins on P- and V-type adenosinetriphosphatases. Biochemistry 32, 3902-3906. doi: 10.1021/bi00066a008

Eck, R., Nguyen, M., Gunther, J., Kunkel, W., and Zipfel, P. F. (2005). The phosphatidylinositol 3-kinase Vps34p of the human pathogenic yeast Candida albicans is a multifunctional protein that interacts with the putative vacuolar H+ -ATPase subunit Vma7p. Int. J. Med. Microbiol. 295, 57-66. doi 10.1016/j.ijmm.2004.12.007

Erickson, T., Liu, L., Gueyikian, A., Zhu, X., Gibbons, J., and Williamson, P. R. (2001). Multiple virulence factors of Cryptococcus neoformans are dependent on VPH1. Mol. Microbiol. 42, 1121-1131. doi: 10.1046/j.1365-2958.2001.02712.x

Finnigan, G. C., Hanson-Smith, V., Stevens, T. H., and Thornton, J. W. (2012) Evolution of increased complexity in a molecular machine. Nature 481, 360-364. doi: $10.1038 /$ nature 10724

Forgac, M. (2007). Vacuolar ATPases: rotary proton pumps in physiology and pathophysiology. Nat. Rev. Mol. Cell Biol. 8, 917-929. doi: 10.1038/nrm2272

Georgopapadakou, N. H., and Walsh, T. J. (1996). Antifungal agents: chemotherapeutic targets and immunologic strategies. Antimicrob. Agents Chemother. 40, 279-291.

Hameed, S., and Fatima, Z. (2013). Novel regulatory mechanisms of pathogenicity and virulence to combat MDR in. Int. J. Microbiol. 2013, 240209. doi $10.1155 / 2013 / 240209$

Hilty, J., Smulian, A. G., and Newman, S. L. (2008). The Histoplasma capsulatum vacuolar ATPase is required for iron homeostasis, intracellular replication in macrophages and virulence in a murine model of histoplasmosis. Mol. Microbiol. 70, 127-139. doi: 10.1111/j.1365-2958.2008.06395.x

Horn, D. L., Neofytos, D., Anaissie, E. J., Fishman, J. A., Steinbach, W. J., Olyaei, A. J., et al. (2009). Epidemiology and outcomes of candidemia in 2019 patients: data from the prospective antifungal therapy alliance registry. Clin. Infect. Dis 48, 1695-1703. doi: 10.1086/599039

Huang, C., and Chang, A. (2011). pH-dependent cargo sorting from the Golgi. J. Biol. Chem. 286, 10058-10065. doi: 10.1074/jbc.M110.197889

Huss, M., and Wieczorek, H. (2009). Inhibitors of V-ATPases: old and new players. J. Exp. Biol. 212, 341-346. doi: 10.1242/jeb.024067

Ibrahim-Granet, O., Philippe, B., Boleti, H., Boisvieux-Ulrich, E., Grenet, D., Stern, M., etal. (2003). Phagocytosis and intracellular fate of Aspergillus fumigatus conidia in alveolar macrophages. Infect. Immun. 71, 891-903. doi: 10.1128/IAI.71.2.891-903.2003

Jefferies, K. C., Cipriano, D. J., and Forgac, M. (2008). Function, structure and regulation of the vacuolar $(\mathrm{H}+)$-ATPases. Arch. Biochem. Biophys. 476, 33-42. doi: $10.1016 /$ j.abb.2008.03.025

Jia, N., Arthington-Skaggs, B., Lee, W., Pierson, C. A., Lees, N. D., Eckstein, J., et al. (2002). Candida albicans sterol C-14 reductase, encoded by the ERG24 gene, as a potential antifungal target site. Antimicrob. Agents Chemother. 46, 947-957. doi 10.1128/AAC.46.4.947-957.2002

Johnson, R. M., Allen, C., Melman, S. D., Waller, A., Young, S. M., Sklar, L. A., et al. (2010). Identification of inhibitors of vacuolar proton-translocating ATPase pumps in yeast by high-throughput screening flow cytometry. Anal. Biochem 398, 203-211. doi: 10.1016/j.ab.2009.12.020

Kane, P. M. (2006). The where, when, and how of organelle acidification by the yeast vacuolar H +-ATPase. Microbiol. Mol. Biol. Rev. 70, 177-191. doi 10.1128/MMBR.70.1.177-191.2006

Kane, P. M. (2007). The long physiological reach of the yeast vacuolar H+-ATPase. J. Bioenerg. Biomembr. 39, 415-421. doi: 10.1007/s10863-007-9112-Z

Kane, P. M. (2012). Targeting reversible disassembly as a mechanism of controlling V-ATPase activity. Curr. Protein Peptide Sci. 13, 117-123. doi: $10.2174 / 138920312800493142$

Kane, P. M., and Parra, K. J. (2000). Assembly and regulation of the yeast vacuolar H(+)-ATPase. J. Exp. Biol. 203, 81-87.

Karkowska-Kuleta, J., Rapala-Kozik, M., and Kozik, A. (2009). Fungi pathogenic to humans: molecular bases of virulence of Candida albicans, Cryptococcus neoformans and Aspergillus fumigatus. Acta Biochim. Pol. 56, 211-224.

Kaur, S., and Mishra, P. (1991). Dimorphism-associated changes in plasma membrane $\mathrm{H}(+)$-ATPase activity of Candida albicans. Arch. Microbiol. 156, 412-415. doi: 10.1007/BF00248719

Kawasaki-Nishi, S., Nishi, T., and Forgac, M. (2001). Yeast V-ATPase complexes containing different isoforms of the $100-\mathrm{kDa}$ a-subunit differ in coupling efficiency and in vivo dissociation. J. Biol. Chem. 276, 17941-17948. doi: 10.1074/jbc.M010790200

Klotz, S. A., Chasin, B. S., Powell, B., Gaur, N. K., and Lipke, P. N. (2007). Polymicrobial bloodstream infections involving Candida species: analysis of patient and review of the literature. Diagn. Microbiol. Infect. Dis. 59, 401-406. doi: 10.1016/j.diagmicrobio.2007.07.001 
Mahanty, S. K., Gupta, P., Banerjee, U., Fotedar, R., and Prasad, R. (1990). Defective plasma membrane $\mathrm{H}(+)$-ATPase or orthovanadate resistant mutants from Candida albicans, a pathogenic yeast. Biochem. Int. 22, 11-20.

Manolson, M. F., Wu, B., Proteau, D., Taillon, B. E., Roberts, B. T., Hoyt, M. A., etal. (1994). STV1 gene encodes functional homologue of 95-kDa yeast vacuolar $\mathrm{H}(+)$-ATPase subunit Vph1p. J. Biol. Chem. 269, 14064 14074.

Marshansky, V., and Futai, M. (2008). The V-type H+-ATPase in vesicular trafficking: targeting, regulation and function. Curr. Opin. Cell Biol. 20, 415-426. doi: 10.1016/j.ceb.2008.03.015

Martinez-Munoz, G. A., and Kane, P. (2008). Vacuolar and plasma membrane proton pumps collaborate to achieve cytosolic $\mathrm{pH}$ homeostasis in yeast. J. Biol. Chem. 283, 20309-20319. doi: 10.1074/jbc.M710470200

Monk, B. C., Kurtz, M. B., Marrinan, J. A., and Perlin, D. S. (1991). Cloning and characterization of the plasma membrane $\mathrm{H}(+)$-ATPase from Candida albicans. J. Bacteriol. 173, 6826-6836.

Monk, B. C., Niimi, M., and Shepherd, M. G. (1993). The Candida albicans plasma membrane and $\mathrm{H}(+)$-ATPase during yeast growth and germ tube formation. $J$. Bacteriol. 175, 5566-5574.

Naglik, J. R., Challacombe, S. J., and Hube, B. (2003). Candida albicans secreted aspartyl proteinases in virulence and pathogenesis. Microbiol. Mol. Biol. Rev. 67, 400-428. doi: 10.1128/MMBR.67.3.400-428.2003

Oot, R. A., and Wilkens, S. (2012). Subunit interactions at the V1-Vo interface in yeast vacuolar ATPase. J. Biol. Chem. 287, 13396-13406. doi: 10.1074/jbc.M112.343962

Palanisamy, S. K., Ramirez, M. A., Lorenz, M., and Lee, S. A. (2010). Candida albicans PEP12 is required for biofilm integrity and in vivo virulence. Eukaryot. Cell. 9, 266-277. doi: 10.1128/EC.00295-299

Papon, N., Courdavault, V., Clastre, M., Simkin, A. J., Creche, J., and GiglioliGuivarc'h, N. (2012). Deus ex Candida genetics: overcoming the hurdles for the development of a molecular toolbox in the CTG clade. Microbiology 158, 585-600. doi: 10.1099/mic.0.055244-55240

Parra, K. J. (2012). "Vacuolar ATPase (V-ATPase) a model proton pump for antifungal drug discovery," in Emerging Strategies for Antimicrobial Drug Discovery, eds G. P. Tegos and E. Mylonakis (Oxfordshire: CABI), 89-100.

Patenaude, C., Zhang, Y., Cormack, B., Kohler, J., and Rao, R. (2013). Essential role for vacuolar acidification in Candida albicans virulence. J. Biol. Chem. 288, 26256-26264. doi: 10.1074/jbc.M113.494815

Perzov, N., Nelson, H., and Nelson, N. (2000). Altered distribution of the yeast plasma membrane $\mathrm{H}+$-ATPase as a feature of vacuolar $\mathrm{H}+$-ATPase null mutants. J. Biol. Chem. 275, 40088-40095. doi: 10.1074/jbc.M007011200

Pfaller, M. A., and Diekema, D. J. (2007). Epidemiology of invasive candidiasis: a persistent public health problem. Clin. Microbiol. Rev. 20, 133-163. doi: 10.1128/CMR.00029-26

Poltermann, S., Nguyen, M., Gunther, J., Wendland, J., Hartl, A., Kunkel, W., et al. (2005). The putative vacuolar ATPase subunit Vma7p of Candida albicans is involved in vacuole acidification, hyphal development and virulence. Microbiology 151, 1645-1655. doi: 10.1099/mic.0.27505-27500

Rahman, S., Yamato, I., Saijo, S., Mizutani, K., Ishizuka-Katsura, Y., Ohsawa, N., et al. (2013). Biochemical and biophysical properties of interactions between subunits of the peripheral stalk region of human V-ATPase. PLoS ONE 8:e55704. doi: 10.1371/journal.pone.0055704

Raines, S. M., Rane, H. S., Bernardo, S. M., Binder, J. L., Lee, S. A., and Parra, K. J. (2013). Deletion of vacuolar proton-translocating ATPase V(o)a isoforms clarifies the role of vacuolar $\mathrm{pH}$ as a determinant of virulence-associated traits in Candida albicans. J. Biol. Chem. 288, 6190-6201. doi: 10.1074/jbc.M112. 426197

Rane, H. S., Bernardo, S. M., Raines, S. M., Binder, J. L., Parra, K. J., and Lee, S. A. (2013). Candida albicans VMA3 is necessary for V-ATPase assembly and function and contributes to secretion and filamentation. Eukaryot. Cell. 12, 1369-1382. doi: 10.1128/EC.00118-113

Slesiona, S., Gressler, M., Mihlan, M., Zaehle, C., Schaller, M., Barz, D., et al. (2012). Persistence versus escape: Aspergillus terreus and Aspergillus fumigatus employ different strategies during interactions with macrophages. PLoS ONE 7:e31223. doi: 10.1371/journal.pone.0031223
Soteropoulos, P., Vaz, T., Santangelo, R., Paderu, P., Huang, D. Y., Tamas, M. J., et al. (2000). Molecular characterization of the plasma membrane $\mathrm{H}(+)$-ATPase, an antifungal target in Cryptococcus neoformans. Antimicrob. Agents Chemother. 44, 2349-2355. doi: 10.1128/AAC.44.9.2349-2355.2000

Stewart, E., Gow, N. A., and Bowen, D. V. (1988). Cytoplasmic alkalinization during germ tube formation in Candida albicans. J. Gen. Microbiol. 134, 1079-1087. doi: 10.1099/00221287-134-5-1079

Stewart, E., Hawser, S., and Gow, N. A. (1989). Changes in internal and external pH accompanying growth of Candida albicans: studies of non-dimorphic variants. Arch. Microbiol. 151, 149-153. doi: 10.1007/BF00414430

Sudbery, P. E. (2011). Growth of Candida albicans hyphae. Nat. Rev. Microbiol. 9 , 737-748. doi: 10.1038/nrmicro2636

Sun-Wada, G. H., and Wada, Y. (2010). Vacuolar-type proton pump ATPases: roles of subunit isoforms in physiology and pathology. Histol. Histopathol. 25 1611-1620.

Sun-Wada, G. H., Wada, Y., and Futai, M. (2003). Vacuolar H+ pumping ATPases in luminal acidic organelles and extracellular compartments: common rotational mechanism and diverse physiological roles. J. Bioenerg. Biomembr. 35, 347-358. doi: 10.1023/A:1025780932403

Tarsio, M., Zheng, H., Smardon, A. M., Martinez-Munoz, G. A., and Kane, P. M. (2011). Consequences of loss of Vph1 protein-containing vacuolar ATPases (VATPases) for overall cellular pH homeostasis. J. Biol. Chem. 286, 28089-28096. doi: 10.1074/jbc.M111.251363

Toei, M., Saum, R., and Forgac, M. (2010). Regulation and isoform function of the V-ATPases. Biochemistry 49, 4715-4723. doi: 10.1021/bi100397s

Ullah, A., Lopes, M. I., Brul, S., and Smits, G. J. (2013). Intracellular pH homeostasis in Candida glabrata in infection-associated conditions. Microbiology 159, 803 813. doi: 10.1099/mic.0.063610-63610

Vanden Bossche, H. (1985). Biochemical targets for antifungal azole derivatives: hypothesis on the mode of action. Curr. Top. Med. Mycol. 1, 313-351. doi: 10.1007/978-1-4613-9547-8_12

Veses, V., Richards, A., and Gow, N. A. (2008). Vacuoles and fungal biology. Curr. Opin. Microbiol. 11, 503-510. doi: 10.1016/j.mib.2008.09.017 doi: 10.1016/j.mib.2008.09.017

Weissman, Z., Shemer, R., Conibear, E., and Kornitzer, D. (2008). An endocytic mechanism for haemoglobin-iron acquisition in Candida albicans. Mol. Microbiol. 69, 201-217. doi: 10.1111/j.1365-2958.2008.06277.x doi: 10.1111/j.13652958.2008.06277.x

Wermuth, C. G. (2004). Selective optimization of side activities: another way for drug discovery. J. Med. Chem. 47, 1303-1314. doi: 10.1021/jm030480f

Wojtowicz, H., Kloc, K., Maliszewska, I., Mlochowski, J., Pietka, M., and Piasecki, E. (2004). Azaanalogues of ebselen as antimicrobial and antiviral agents: synthesis and properties. Farmaco 59, 863-868. doi: 10.1016/j.farmac.2004.07.003

Zhang, Y. Q., Gamarra, S., Garcia-Effron, G., Park, S., Perlin, D. S., and Rao, R. (2010). Requirement for ergosterol in V-ATPase function underlies antifungal activity of azole drugs. PLoS Pathog. 6:e1000939. doi: 10.1371/journal.ppat.1000939

Conflict of Interest Statement: The authors declare that the research was conducted in the absence of any commercial or financial relationships that could be construed as a potential conflict of interest.

Received: 03 November 2013; accepted: 06 January 2014; published online: 27 January 2014.

Citation: Hayek SR, Lee SA and Parra KJ (2014) Advances in targeting the vacuolar proton-translocating ATPase (V-ATPase) for anti-fungal therapy. Front. Pharmacol. 5:4. doi: 10.3389/fphar.2014.00004

This article was submitted to Experimental Pharmacology and Drug Discovery, a section of the journal Frontiers in Pharmacology.

Copyright $(2) 2014$ Hayek, Lee and Parra. This is an open-access article distributed under the terms of the Creative Commons Attribution License (CC BY). The use, distribution or reproduction in other forums is permitted, provided the original author (s) or licensor are credited and that the original publication in this journal is cited, in accordance with accepted academic practice. No use, distribution or reproduction is permitted which does not comply with these terms. 